\title{
More About Four Biquadrates Equal One Biquadrate
}

\author{
By Kermit Rose and Simcha Brudno
}

\begin{abstract}
A computer-generated table of the first 82 numerical solutions of $A^{4}+B^{4}+$ $C^{4}+D^{4}=E^{4}$ is presented. Some regularities are noted.
\end{abstract}

Solutions of the Diophantine equation $A^{4}+B^{4}+C^{4}+D^{4}=E^{4}$ in least integers have been obtained by several authors [1] [6]. The following table includes the first 82 consecutive primitive solutions of $A^{4}+B^{4}+C^{4}+D^{4}=E^{4}$. A primitive solution is one for which the GCD of $A, B, C, D$ and $E$ equals 1 . The method used to find these numerical solutions combines those of Leech [5], Ward [3] and Brudno [6].

In addition, the authors used the following new restrictions:

(1) The sum of two fourth powers cannot be 7, 8, or 11 (Mod 13);

(2) The sum of two fourth powers cannot be 6, 7, 10, or $11(\operatorname{Mod} 17)$;

(3) The sum of two fourth powers cannot be 4, 5, 6, 9, 13, 22 or 28 (Mod 29);

(4) The sum of three fourth powers cannot be zero modulo 29 unless each fourth power is zero modulo 29 . The same applies to modulo 16 and modulo 5 .

Some of these restrictions were used by Lander [4] in his search for solutions to $A^{4}=B^{4}+C^{4}+D^{4}$.

The table is divided into seven columns where the first three are the $A_{i}, B_{i}, C_{i}=$ $0(\operatorname{Mod} 5)$. The fourth column is the $D_{i} \neq 0(\operatorname{Mod} 5)$; the fifth is the appropriate $E_{i}$ where $A_{i}^{4}+B_{i}^{4}+C_{i}^{4}+D_{i}^{4}=E_{i}^{4}$. The sixth column contains an index for each $E_{i}$. The following table explains the index:

$$
\begin{array}{cl}
\text { Index } & \multicolumn{1}{c}{\text { Restriction on } D} \\
0 & E-D=0\left(\operatorname{Mod} 5^{4}\right) \\
1 & 182 E-D=0\left(\operatorname{Mod} 5^{4}\right) \\
2 & E+D=0\left(\operatorname{Mod} 5^{4}\right) \\
3 & 182 E+D=0\left(\operatorname{Mod} 5^{4}\right)
\end{array}
$$

$$
\begin{array}{ll} 
& \text { Equivalent Formulations } \\
E=P+625 Q, \quad D=P-625 Q \\
E=24 P+7 Q, \quad D=24 Q-7 P \\
E=625 P+Q, \quad D=625 P-Q \\
E=24 P-7 Q, \quad D=7 P+24 Q
\end{array}
$$

The last column gives a sequence number of the solution. The first 23 solutions were already given elsewhere (see [4]). The last 6 solutions are not consecutive and therefore the numbering sequence stops at 82 .

There is a regularity observed in cases 24 to 29 , inclusive. They result in a "double triplet" of the $E_{i}$ with $\Delta 60$. Furthermore, cases 49 and 63 are related by having the same $P=616$ and $Q=225$. The numbers were calculated using a CDC 6400 computer at Florida State University, Tallahassee, Florida, and a 7094 at M. I. T., Cambridge, Massachusetts.

Conjecture. Every Diophantine equation of the form

$$
\sum_{i=1}^{p-1} X_{i}^{p-1}=y^{p-1}, \quad p \text { a prime }
$$

Received April 26, 1972.

AMS (MOS) subject classifications (1970). Primary 10-04, 10B15.

Key words and phrases. Fourth-order equations, numerical tables. 
TABle 1

$\begin{array}{llllll}A_{i} & B_{i} & C_{i} & D_{i} & E_{i} & \text { Index }\end{array}$

\begin{tabular}{|c|c|c|c|c|c|c|}
\hline$A$ & $B$ & $C$ & $D$ & $E$ & Index & $N R$ \\
\hline 30 & 120 & 315 & 272 & 353 & 2 & 1 \\
\hline 240 & 340 & 430 & 599 & 651 & 2 & 2 \\
\hline 2420 & 710 & 435 & 1384 & 2487 & 1 & 3 \\
\hline 2365 & 1190 & 1130 & 1432 & 2501 & 1 & 4 \\
\hline 2745 & 1010 & 850 & 1546 & 2829 & 2 & 5 \\
\hline 2460 & 2345 & 2270 & 3152 & 3723 & 2 & 6 \\
\hline 3395 & 3230 & 350 & 1652 & 3973 & 2 & 7 \\
\hline 2650 & 1060 & 205 & 4094 & 4267 & 1 & 8 \\
\hline 3670 & 3545 & 1750 & 1394 & 4333 & 3 & 9 \\
\hline 4250 & 2840 & 700 & 699 & 4449 & 0 & 10 \\
\hline 1880 & 1660 & 380 & 4907 & 4949 & 3 & 11 \\
\hline 5080 & 1120 & 1000 & 3233 & 5281 & 3 & 12 \\
\hline 5055 & 3910 & 410 & 1412 & 5463 & 2 & 13 \\
\hline 5400 & 1770 & 955 & 2634 & 5491 & 2 & 14 \\
\hline 5400 & 1680 & 30 & 3043 & 5543 & 0 & 15 \\
\hline 5150 & 4355 & 1810 & 1354 & 5729 & 0 & 16 \\
\hline 5695 & 4280 & 2770 & 542 & 6167 & 0 & 17 \\
\hline 5000 & 885 & 50 & 5984 & 6609 & 0 & 18 \\
\hline 6185 & 4790 & 1490 & 3468 & 6801 & 3 & 19 \\
\hline 5365 & 2850 & 1390 & 6368 & 7101 & 3 & 20 \\
\hline 2790 & 1345 & 160 & 7166 & 7209 & 2 & 21 \\
\hline 6635 & 5440 & 800 & 3052 & 7339 & 3 & 22 \\
\hline 6995 & 5620 & 2230 & 3196 & 7703 & 1 & 23 \\
\hline 5670 & 5500 & 4450 & 7123 & 8373 & 0 & 24 \\
\hline 7565 & 5230 & 4730 & 4806 & 8433 & 1 & 25 \\
\hline 7630 & 5925 & 4910 & 524 & 8493 & 3 & 26 \\
\hline 7815 & 6100 & 3440 & 1642 & 8517 & 0 & 27 \\
\hline 8230 & 2905 & 1050 & 5236 & 8577 & 3 & 28 \\
\hline 5780 & 3695 & 3450 & 8012 & 8637 & 0 & 29 \\
\hline 8570 & 6180 & 3285 & 816 & 9137 & 3 & 30 \\
\hline 6435 & 2870 & 680 & 8618 & 9243 & 0 & 31 \\
\hline 7820 & 6935 & 5800 & 5192 & 9431 & 1 & 32 \\
\hline 8760 & 6935 & 1490 & 1394 & 9519 & 0 & 33 \\
\hline 8570 & 7050 & 305 & 5264 & 9639 & 0 & 34 \\
\hline 8835 & 6800 & 5490 & 2922 & 9797 & 0 & 35 \\
\hline 6485 & 5660 & 4840 & 8864 & 9877 & 1 & 36 \\
\hline 8870 & 8635 & 1620 & 2294 & 10419 & 0 & 37 \\
\hline 9145 & 8530 & 5300 & 5936 & 10939 & 2 & 38 \\
\hline 10490 & 8635 & 5300 & 3556 & 11681 & 0 & 39 \\
\hline 11455 & 6200 & 4490 & 1476 & 11757 & 3 & 40 \\
\hline
\end{tabular}


TABLE 1 (Continued)

$A_{i} \quad B_{i} \quad C_{i} \quad D_{i} \quad E_{i}$ Index

\begin{tabular}{|c|c|c|c|c|c|c|}
\hline$A$ & $B$ & $C$ & $D$ & $E$ & Index & $N R$ \\
\hline 8735 & 8170 & 1180 & 10144 & 12019 & 0 & 41 \\
\hline 11720 & 7270 & 3710 & 2833 & 12167 & 2 & 42 \\
\hline 9360 & 8655 & 7480 & 8862 & 12259 & 3 & 43 \\
\hline 8925 & 4410 & 3450 & 11234 & 12287 & 1 & 44 \\
\hline 11390 & 8045 & 320 & 7352 & 12439 & 3 & 45 \\
\hline 12435 & 6190 & 5780 & 1616 & 12759 & 2 & 46 \\
\hline 10310 & 6870 & 2935 & 10678 & 12771 & 3 & 47 \\
\hline 12845 & 5950 & 2870 & 5934 & 13137 & 1 & 48 \\
\hline 11210 & 7590 & 7025 & 9712 & 13209 & 3 & 49 \\
\hline 13040 & 4975 & 1700 & 7896 & 13521 & 0 & 50 \\
\hline 12035 & 3610 & 3440 & 10738 & 13637 & 2 & 51 \\
\hline 13410 & 6420 & 1275 & 8278 & 14029 & 1 & 52 \\
\hline 13740 & 7920 & 6660 & 3929 & 14297 & 1 & 53 \\
\hline 14405 & 2630 & 210 & 34 & 14409 & 0 & 54 \\
\hline 13355 & 8010 & 1530 & 9498 & 14489 & 1 & 55 \\
\hline 13900 & 2040 & 1920 & 9219 & 14531 & 2 & 56 \\
\hline 13760 & 10245 & 800 & 4682 & 14751 & 1 & 57 \\
\hline 14815 & 8940 & 4250 & 2512 & 15309 & 3 & 58 \\
\hline 11110 & 6800 & 3890 & 14579 & 15829 & 0 & 59 \\
\hline 11815 & 5640 & 2880 & 14598 & 16027 & 2 & 60 \\
\hline 15780 & 4790 & 4140 & 7701 & 16049 & 2 & 61 \\
\hline 15940 & 6670 & 5430 & 137 & 16113 & 2 & 62 \\
\hline 14320 & 13110 & 2275 & 1088 & 16359 & 1 & 63 \\
\hline 14890 & 8830 & 1220 & 12107 & 16643 & 2 & 64 \\
\hline 15160 & 11015 & 10850 & 412 & 16891 & 1 & 65 \\
\hline 11810 & 2350 & 1845 & 15776 & 16893 & 1 & 66 \\
\hline 15375 & 11050 & 6690 & 11658 & 17381 & 3 & 67 \\
\hline 13060 & 8495 & 1220 & 15644 & 17519 & 0 & 68 \\
\hline 16405 & 6500 & 950 & 11896 & 17521 & 0 & 69 \\
\hline 16215 & 12850 & 5450 & 1802 & 17661 & 1 & 70 \\
\hline 10660 & 3235 & 3220 & 17068 & 17693 & 0 & 71 \\
\hline 17320 & 9860 & 1945 & 7256 & 17881 & 0 & 72 \\
\hline 17510 & 8340 & 2760 & 9423 & 18077 & 2 & 73 \\
\hline 16805 & 13660 & 5270 & 5898 & 18477 & 2 & 74 \\
\hline 15365 & 12430 & 11410 & 12668 & 18701 & 3 & 75 \\
\hline 16560 & 8355 & 610 & 15906 & 19483 & 1 & 76 \\
\hline 13940 & 9305 & 4460 & 17726 & 19493 & 1 & 77 \\
\hline 17595 & 13440 & 5370 & 12772 & 19871 & 1 & 78 \\
\hline 19255 & 3090 & 780 & 12702 & 20111 & 1 & 79 \\
\hline 11980 & 8975 & 1090 & 19244 & 20131 & 2 & 80 \\
\hline
\end{tabular}


TABLE 1 (Continued)

$A_{i} \quad B_{i} \quad C_{i} \quad D_{i} \quad E_{i} \quad$ Index

\begin{tabular}{crrrccr}
\hline $\boldsymbol{A}$ & \multicolumn{1}{c}{$\boldsymbol{B}$} & \multicolumn{1}{c}{$\boldsymbol{C}$} & \multicolumn{1}{c}{$\boldsymbol{D}$} & $\boldsymbol{E}$ & Index & $N R$ \\
\hline 19670 & 10030 & 1880 & 9579 & 20253 & 3 & 81 \\
19480 & 7550 & 1660 & 12969 & 20469 & 0 & 82 \\
18100 & 13690 & 12140 & 11801 & 20699 & 2 & \\
13970 & 8855 & 8720 & 19142 & 20719 & 3 & \\
17740 & 16525 & 12070 & 3362 & 21013 & 2 & \\
13915 & 5950 & 5420 & 24802 & 25427 & 0 & \\
16260 & 12860 & 8545 & 34178 & 34803 & 0 & \\
1840 & 30690 & 41000 & 89929 & 91179 & 0 & \\
\hline
\end{tabular}

has at least one solution in integers such that $x+y=p^{p-1}$ where $x$ is one of the $x_{i}$.

The known examples are

$$
\begin{gathered}
p=2 . \\
1^{1}=1^{1} ; \quad 1+1=2 . \\
p=3 . \\
3^{2}+4^{2}=5^{2} ; \quad 4+5=3^{2} . \\
\\
\quad 30^{4}+120^{4}+272^{4}+315^{4}=353^{4} ; \quad 272+353=5^{4} .
\end{gathered}
$$

For $p=7$ no solution is known. The problem of six integers to the sixth power equal to one to the sixth was attacked [4, pp. 454-455] and a computer search for up to $y=38314$ was unfortunately negative.

However, as indicated by this conjecture, a possible solution may be found for $y$ greater than $58825\left(=7^{6} / 2+1\right)$.

Mathematics Department

Florida State University

Tallahassee, Florida 32306

The Medical Center

University of Illinois

835 South Wolcott Street

Room E-6, MSA Building

Chicago, Illinois 60612

1. R. Norrie, University of St. Andrews 500th Anniversary Memorial Volume, Edinburgh, 1911.

2. J. O. Patterson, "A note on the Diophantine problem of finding four biquadrates whose sum is a biquadrate," Bull., Amer. Math. Soc., v. 48, 1942, pp. 736-737. MR 4, 33.

3. M. WARD, "Euler's problem on sums of three fourth powers," Duke Math. J., v. 15, 1948, pp. 827-837. MR 10, 283.

4. L. J. Lander, T. R. Parkin \& J. L. Selfridge, "A survey of equal sums of like powers," Math. Comp., v. 21, 1967, pp. 446-459. MR 36 \#5060.

5. J. LeEch, "On $A^{4}+B^{4}+C^{4}+D^{4}=E^{4}$," Proc. Cambridge Philos. Soc., v. 54, 1958, pp. 554-555. MR $20 \# 2301$.

6. S. BRudno, "A further example of $A^{4}+B^{4}+C^{4}+D^{4}=E^{4}$," Proc. Cambridge Philos. Soc., v. 60, 1964, pp. 1027-1028. MR 29 \#329. 